\title{
Low-Surfactant Microemulsions for Enhanced Topical Delivery of Poorly Soluble Drugs
}

\author{
Alireza Shalviri ${ }^{1}$, Avinash Sharma ${ }^{2}$, Dipak Patel ${ }^{2}$ and Amyn Sayani ${ }^{2}$ \\ ${ }^{1}$ Faculty of Pharmacy, University of Toronto, Toronto, Ontario. \\ ${ }^{2}$ Pharmaceutical Development Department, GlaxoSmithKline Inc., Mississauga Road North, Mississauga, Ontario.
}

Received, January 18, 2011; Revised, May 27, 2011; Accepted, July 26, 2011; Published, July 31, 2011.

\begin{abstract}
Purpose: To develop and characterize low-surfactant microemulsion (ME) gels to enhance topical delivery of poorly soluble drugs. Method: Five low surfactant ME formulations were manufactured following the construction of pseudo-ternary phase diagrams. The MEs were screened for their ability to dissolve a poorly soluble new chemical entity (Model Drug X). Various viscosity imparting agents like Carbopol 934, Colloidal Silica, HPMC K100M, Lubrajel NP, and Xanthan Gum were evaluated for the manufacture of these ME gels. Each ME gel was then further evaluated for physical stability, including assessing rheological profiles. In vitro release profiles were also determined and compared to a conventional ointment. Results: Three of the five low surfactant MEs developed (ME1, ME4 and ME5) were capable of dissolving Model Drug X up to 14 fold higher than the conventional ointment formulation. ME1 and ME4 gels comprising Xanthan gum or Carbopol 934 were physically stable, while ME5 gel was stable only with Colloidal Silica. The ME5 gel with Colloidal Silica showed an irreversible increase in its elastic modulus when exposed to high temperature, indicating that the formulation would be less suitable for commercial use. The Xanthan Gum and Colloidal Silica gels yielded significantly higher release rates ( 8 - 10 fold) compared to a conventional ointment and formulations containing Carbopol 934. The significant difference in drug release rates between Xanthan Gum and Carbopol 934 indicated that choice of viscosity imparting agent played an important role in governing drug release from ME gels. Conclusion: ME gels were developed with low surfactant concentrations and improved formulation characteristics, which increased the solubility and subsequent release of a poorly soluble drug.
\end{abstract}

This article is open to POST-PUBLICATION REVIEW. Registered readers (see "For Readers") may comment by clicking on ABSTRACT on the issue's contents page.

\section{INTRODUCTION}

Microemulsions (MEs) are systems of water, with or without electrolyte, oil and surfactant(s) that are transparent and thermodynamically stable with the potential for enhancing drug solubility as well as drug penetration (1). The main difference between a conventional emulsion and a ME is the droplet size of the dispersed phase. A conventional emulsion has droplet size in the range of $100-100,000 \mathrm{~nm}$ and hence is turbid in appearance. In a ME, the droplet size of the dispersed phase is smaller than one fourth the wavelength of visible light, usually below $140 \mathrm{~nm}$ in diameter, which makes MEs transparent liquids (2). Although there is an overlap of the globule size ranges for these two types of emulsions, the conventional emulsions may have, if at all, only a limited number of globules below $140 \mathrm{~nm}$.
MEs offer enhanced solubilization for poorly water soluble drugs, which can make them superior drug delivery systems compared to conventional emulsions. Solubilization of a wide range of drugs is due to the presence of large fractions of lipophilic and hydrophilic phases and their unique structural organization of these phases (3-5). In addition, recent studies indicate that the non-ionic surfactants used in these formulations can affect the skin barrier function to potentially increase the drug penetration into the skin $(6,7)$.

Corresponding Author: Avinash Sharma; GlaxoSmithKline Inc., Mississauga Road North, Mississauga, Ontario, Canada; E-Mail: avinash.c.sharma@gsk.com 
Unlike conventional emulsions, the MEs are generally prepared by simple mixing of appropriate quantities of the components at ambient temperature. Their transparency and low viscosity make the filtration process and inspection of foreign particles easy if these formulations are used for parenteral use (3). These properties have obvious implications when considering the relative commercialization costs of the two systems.

Despite their favourable properties, MEs have not enjoyed wide commercial success, with only a few commercial products on the market. One reason for their limited use likely stems from the narrow range of pharmaceutically acceptable surfactants and co-surfactants available for designing ME formulations. In addition, high concentrations of surfactants, often required for their formation, can result in skin irritation.

Use of ME for topical application is also limited by their low viscosity; therefore, viscosity imparting agents are required to increase the viscosity of formulations (8). Determining a proper viscosity imparting agent can be difficult since modification of the rheological behaviour of the formulations can negatively impact ME stability, drug release rates and the large water/oil interface. An ideal polymer must be soluble in the continuous phase to display non-covalent intermolecular interactions, which act cooperatively and lead to the formation of a polymer network (8). Further, the polymers must be biocompatible and exhibit few interactions with surfactants and other formulation excipients in order to be pharmaceutically acceptable $(9,10)$.

The objective of this research was to develop topical ME gels for enhanced topical delivery of a poorly soluble model drug with low surfactant concentrations to minimize skin irritation.

\section{MATERIALS AND METHODS}

\section{Materials}

Tetraglycol, Tween 80 (Polysorbate 80), Brij 30 (Polyoxylethylene-4-LaurylEther), Triethanolamine and Oleic acid were obtained from Sigma-Aldrich, Canada. Labrasol (Caprylocaproyl Macroglycerides), Isostearyl Isostearate, Plurol diisostearique (Polyglyceryl diisostearate), Transcutol P (Diethyleneglycol monoethylether) were received from Gattefosse, Canada. Miglyol
812 (Caprylic/Capric Triglycerides) was received from Croda, Canada. Carbopol 934 (Carbomer) was obtained from Noveon, Canada. Isopropyl Myristate (IPM) was obtained from Uniquema. Propylene glycol and Hydroxypropyl Methylcellulose K100M were obtained from Dow chemical, Canada, and Cabosil (Colloidal Silicon Dioxide) was obtained from Cabot Corporation, Xantural 180 (Xanthan Gum) was obtained from CP Kelco and Lubrajel NP (Glycerine and Glyceryl Acrylate/Acrylic Acid copolymers) was received as a sample from United Guardian Inc. A poorly soluble new chemical entity from GSK's portfolio with M. Wt. 474, $\log \mathrm{P} 3.65$ and practically insoluble in water was used as a model drug (Drug $\mathrm{X})$.

\section{Drug Solubility}

The solubility of a model NCE, Drug X, was assessed visually in individual components and MEs. Approximately $5 \mathrm{mg}$ of drug was weighed in a $10 \times 75 \mathrm{~mm}$ test tube. The drug was then mixed with increasingly larger amounts of solvents using Fisher vortex for one minute. The solutions were sonicated, if required, for 1-2 minutes to reduce foam and air bubbles produced during vortexing. A maximum of 8 grams of solvent was used for dissolving approximately $5 \mathrm{mg}$ of Drug X. The drug solutions obtained were visually inspected for signs of drug crystallization and precipitation at 20 minutes, $24 \mathrm{~h}, 48 \mathrm{~h}$, and $72 \mathrm{~h}$. Solubility $(\mathrm{mg} / \mathrm{g})$ was calculated by dividing the weight of drug $(\mathrm{mg})$ by the weight of solvent $(\mathrm{g})$ which was required to obtain a clear solution after $72 \mathrm{~h}$.

\section{Construction of Pseudo-Ternary Phase Diagrams}

Pseudo-ternary phase diagrams were prepared by mixing different amounts of oil with a fixed ratio of surfactant(s)/co-surfactant(s) and then adding water drop wise with gentle stirring at room temperature. After being equilibrated, the mixtures were visually assessed for transparency to determine if the resulting system was a ME or a conventional emulsion. No attempt was made to distinguish between oil-in-water, water-in-oil or bicontinuous type MEs. 


\section{Manufacture of Microemulsions Containing Drug X}

For the preparation of microemulsions containing the active, a desired amount of the drug was dissolved in the aqueous phase (Water/Propylene Glycol), while stirring, to obtain $0.5 \%(\mathrm{w} / \mathrm{w})$ drug loading. This drug solution was subsequently added to the appropriate mixture of oil and surfactant(s) and mixed for 10 minutes using a magnetic stirrer. In ME5, which does not contain PG, the drug could not be completely dissolved in aqueous phase and was added directly to the prepared ME followed by mixing for 10 minutes to dissolve it completely.

\section{Microemulsion Gels}

Xanthan Gum, Carbopol 934, Lubrajel NP, Colloidal Silica, and HPMC K100M were evaluated in various concentrations for their ability to impart viscosity to the ME formulations. The viscosity imparting agent was added to the ME and mixed with a magnetic stirrer until a uniform dispersion was achieved. The ME gels were inspected visually after $24 \mathrm{~h}$ at room temperature for phase separation or turbidity. Carbopol 934 gel was adjusted to $\mathrm{pH}$ 4.5 to 6.5 with Triethanolamine.

\section{Stability Studies}

Accelerated testing for physical stability of the ME gels was conducted by storing samples in an environmentally controlled stability chamber at $40^{\circ} \mathrm{C} / 75 \%$ for 2 months. The samples were visually inspected on a weekly basis for their physical appearance and any sign of turbidity and phase separation. Temperature cycling was conducted over 14 days starting at $5^{\circ} \mathrm{C}$ for $24 \mathrm{~h}$ and then increasing to $40^{\circ} \mathrm{C}$ for $24 \mathrm{~h}$. The cycle was repeated over 13 days. The final cycle was set at room temperature for $24 \mathrm{~h}$.

ME gels were centrifuged at $4000 \mathrm{rpm}$ and $25^{\circ} \mathrm{C}$ for 60 minutes using Eppendorf Centrifuge $5810 \mathrm{R}$.

\section{Rheological Assessments}

The rheological behaviour of all ME gels formulations was assessed using a Dynamic Stress Rheometer SR5000 equipped with Peltier environmental system. A $40 \mathrm{~mm}$ parallel plate with gap of $0.6 \mathrm{~mm}$ was used to perform dynamic frequency sweep and temperature ramp tests at frequency of $6.28 \mathrm{rad} / \mathrm{s}$. Mineral oil was used to coat the edges of samples to prevent evaporation of water for tests with long duration. The following is brief description for each measurement conducted in this study.

1) Processability test: To predict flow properties of ME gels under different processing conditions (e.g. pumping, mixing, and bottling) and during application on the skin, dynamic stress sweep tests were performed at $25^{\circ} \mathrm{C}$ and $32^{\circ} \mathrm{C}$ respectively.

2) Thermal stability tests:

a. Temperature ramp cycle: The temperature was changed between $5^{\circ} \mathrm{C}$ and $45^{\circ} \mathrm{C}$ for 3 cycles with heating rate of $2^{\circ} \mathrm{C} / \mathrm{min}$ to simulate temperature variations in weather in different places and seasons (11).

b. Temperature step test: The test simulates temperature changes in storage and transportation (11). First, temperature was kept at $25^{\circ} \mathrm{C}$ for 500 seconds; next it was increased quickly to $50^{\circ} \mathrm{C}$ at the ramp rate of $10^{\circ} \mathrm{C} / \mathrm{min}$, and was maintained for 2000 seconds. Finally the temperature was returned to $25^{\circ} \mathrm{C}$ at rate of $10^{\circ} \mathrm{C} / \mathrm{min}$ and maintained for 800 seconds.

\section{In Vitro Release Study}

The in vitro release rates of Drug $\mathrm{X}$ from $\mathrm{ME}$ gel formulations were investigated using Hanson MicroettePlus Diffusion Cell System, with average diffusion area of $1.8 \mathrm{~cm}^{2}$ and average cell volume of $6.6 \mathrm{~mL}$. The receptor compartments were filled with $30 \%$ ethanol and $70 \%$ water. The receptor media was maintained at skin surface temperature of $32^{\circ} \mathrm{C}$ using a re-circulating water bath, and was stirred by externally driven Teflon coated magnetic bars. Supported plain membranes, $0.45 \mu \mathrm{m}$ Acetate Plus, were soaked in the receptor media for 30 minutes before use. Approximately 1-1.5 g of the formulations containing $0.5 \%$ active were applied to the donor cells and then clamped onto the receiver cells with the membrane in between the donor and the receiver cells. Samples $(2.5 \mathrm{~mL})$ were collected for analysis at defined time intervals $(0.5 \mathrm{~h}, 1 \mathrm{~h}, 2 \mathrm{~h}, 4 \mathrm{~h}$, and $6 \mathrm{~h})$ and replaced immediately with fresh receptor media. The 
samples were then analyzed using a highperformance liquid chromatography method.

Two sets of data were collected for each formulation. The cumulative amount of drug released per surface area (Q) was calculated using the following equation (11):

$$
\mathrm{Q}=\frac{C_{n} V+\left(\sum_{i=1}^{i=n-1} C_{i}\right) S}{A}
$$

Where Q $\left(\mu \mathrm{g} / \mathrm{cm}^{2}\right)$ is the cumulative amount of drug released per surface area, $C_{n}(\mu \mathrm{g} / \mathrm{mL})$ is the drug concentration of the receiver solution at $\mathrm{n}^{\text {th }}$ interval, $\mathrm{V}(\mathrm{mL})$ is the volume of the diffusion cell, $\mathrm{C}_{\mathrm{i}}$ $(\mu \mathrm{g} / \mathrm{mL})$ is the sum of concentrations determined for sampling intervals 1 through $\mathrm{n}-1, \mathrm{~S}(\mathrm{~mL})$ is volume of the sample aliquot, and $\mathrm{A}\left(\mathrm{cm}^{2}\right)$ is the diffusion area. A plot of Q vs. square root of time was constructed and the release rate (Js, $\mu \mathrm{g} / \mathrm{cm}^{2} / \mathrm{h}^{0.5}$ ) was determined by linear regression.

\section{RESULTS}

\section{Microemulsion Formulation Development}

Various solvents, surfactants and oils were screened for solubility of Drug X for ME development. The solubility results are shown in Table 1 . The data shows that solubility was highest in Labrasol, Brij 30 and Tween 80 (surfactants); and Tetraglycol, Propylene Glycol and Transcutol P (water miscible solvents). If $5 \mathrm{mg}$ of Drug X could not be dissolved in $8 \mathrm{~g}$ of solvents e.g. for oils and water, the solubility was expressed as $<0.6 \mathrm{mg} / \mathrm{mL}$.

Pseudo-ternary phase diagrams were constructed for different oils using surfactant systems that had the highest solubility of Drug X. The Figure 1 (A C) shows the pseudoternary phase diagrams of the three MEs that were progressed. The shaded areas represent the region of existence for MEs. Any point within the region of existence represents concentrations of three phases required to form a ME formulation.

\begin{tabular}{lc}
\hline $\begin{array}{l}\text { Table 1. Solubility of Drug X in } \\
\text { components. }\end{array}$ & \\
\hline Ingredient & Solubility(mg/g) \\
\hline Miglyol & $<0.6$ \\
Isostearyl Isostearate & $<0.6$ \\
Oleic Acid & $<0.6$ \\
Isopropyl Myristate & $<0.6$ \\
Labrasol & 5.01 \\
Brij 30 & 4.23 \\
Tween 80 & 5.83 \\
Tetraglycol & 1.22 \\
Propylene Glycol & 5.03 \\
Trascutol P & 5.01 \\
Water & $<0.6$ \\
Propylene Glycol /Water $(1: 1)$ & 5.34 \\
\hline
\end{tabular}

Phase diagrams for ME2 and ME3 are not shown because the solubility of the drug in these MEs was $<0.6 \mathrm{mg} / \mathrm{g}$. For ME1, the ternary phase diagram ( Figure $1 \mathrm{~A}$ ) shows that it has a relatively small region of existence. It is possible that the large molecular volume of Miglyol is preventing the close packing of the oil molecules in aqueous phase and limiting the size and stability of oil globules (14). ME5 is also unique as it allows for highest concentration of oil phase to be incorporated into the system (Figure $1 \mathrm{C}$ ). This would be advantageous when dealing with highly oil soluble drug.

Using the regions of existence from the pseudoternary phase diagrams, low surfactant MEs were further developed. In some cases the concentrations of solvents and co-solvents were altered to obtain higher drug solubility. The composition of these formulations is given in Table 2. The conventional ointment formulation contained White petrolatum $(70 \% \mathrm{w} / \mathrm{w})$, Mineral Oil $(5 \% \mathrm{w} / \mathrm{w})$, Polyoxyl 2 Stearyl Ether $(5 \% \mathrm{w} / \mathrm{w})$, Propylene Glycol $(20 \% \mathrm{w} / \mathrm{w})$.

\section{Microemulsion Screening}

The ME formulations (ME1 - ME5) remained clear and isotropic when tested for physical stability at accelerated conditions $\left(40^{\circ} \mathrm{C}\right)$ for 2 months and after temperature cycling over 14 days.

The ME formulations were further screened for solubility of model drug $X$. The results shown in Table 3 indicate that ME1, ME4 and ME5 could dissolve drug $\mathrm{X}, 8$ to 14 fold higher compared to ME2, ME3 and conventional ointment. 
A: Region of existence for ME1

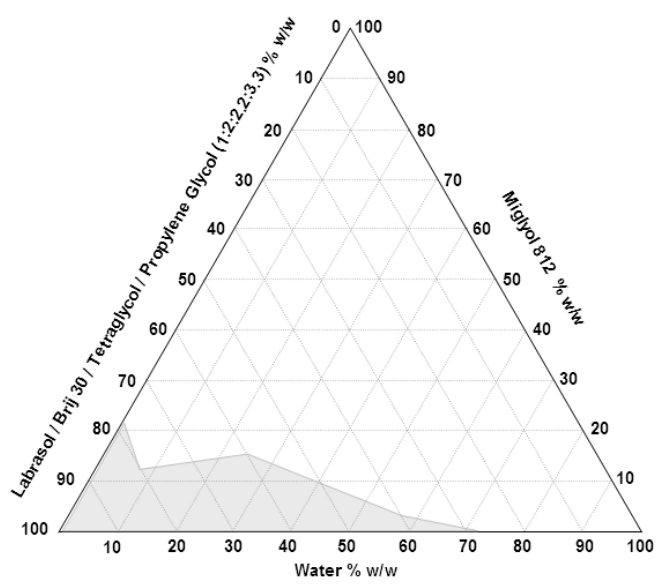

B: Region of existence for ME4

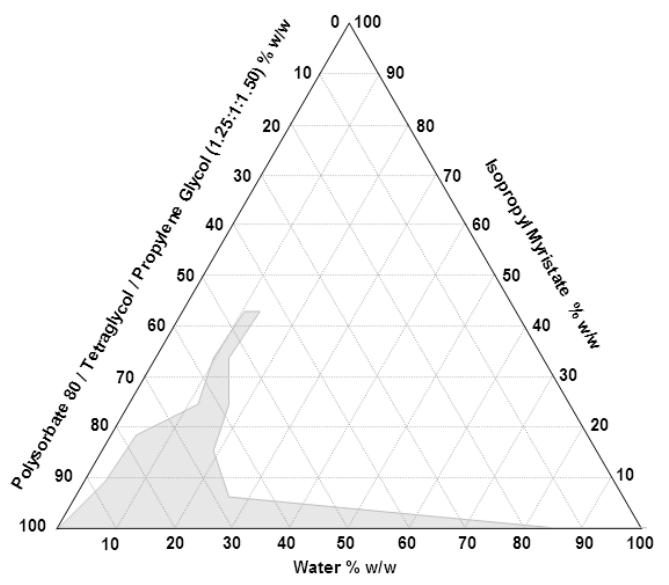

C: Region of existence for ME5

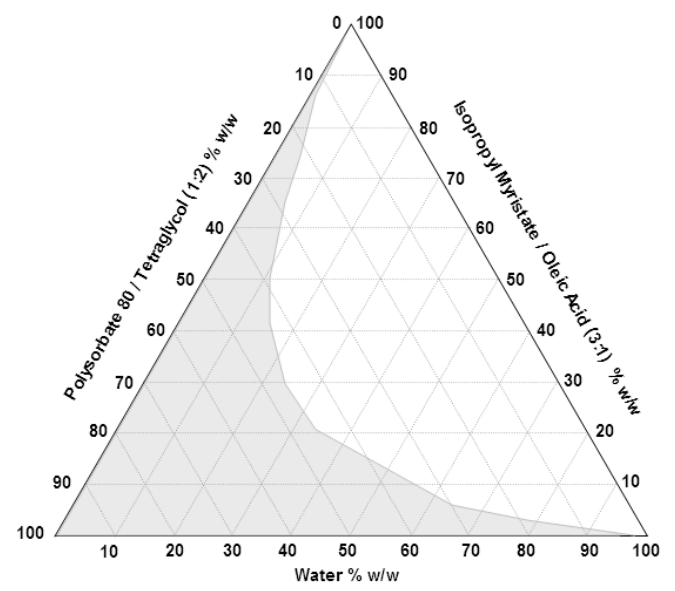

Figure 1. Pseudo-ternary phase diagrams for three MEs showing region of existence

\begin{tabular}{lccccc}
\hline Table 2. Compositions of the MEs investigated \\
\hline & \multicolumn{5}{c}{ \% w/w } \\
\cline { 2 - 6 } Components & ME & ME & ME & ME & ME \\
& $\mathbf{1}$ & $\mathbf{2}$ & $\mathbf{3}$ & $\mathbf{4}$ & $\mathbf{5}$ \\
\hline Miglyol 812 & 5 & - & - & - & - \\
Labrasol & 9 & 25 & 17.5 & - & - \\
Brij 30 & 17 & - & - & - & - \\
Tetraglycol & 7.5 & - & - & 16 & 31 \\
Propylene & 37.5 & - & - & 29 & - \\
Glycol & & & & & \\
Isostearyl & - & 8 & 10 & - & - \\
Isostearate & & & & & \\
Plurol Diiso- & - & 12 & 33 & - & - \\
stearique & & & & & \\
Transcutol P & - & - & 19.5 & - & - \\
Isopropyl & - & - & - & 6.5 & 28.5 \\
Myristate & & & & & \\
Oleic Acid & - & - & - & - & 9 \\
Tween 80 & - & - & - & 25.5 & 16.5 \\
Purified Water & 24 & 55 & 20 & 23 & 15 \\
\hline
\end{tabular}

\begin{tabular}{cc}
\hline Table 3. Solubility of drug X & in microemulsions \\
\hline Microemulsion & Solubility (mg/g) \\
\hline ME1 & 14.1 \\
ME2 & $<0.6$ \\
ME3 & $<0.6$ \\
ME4 & 8.62 \\
ME5 & 10.1 \\
Conventional Ointment & 1.0 \\
\hline
\end{tabular}

Solubility of drug $\mathrm{X}$ in the ME1, ME4 and ME5 was also greater than in the individual excipients at the concentrations used in formulation. This may be due to the synergistic effect of surfactants, water and co-solvents. However, an opposite effect was observed in ME2 and ME3, where the combination of excipients resulted in marked decrease in solubility compared to the added solubility in individual excipients. Based on these solubility results ME1, ME4 and ME5 were selected for further development.

\section{Microemulsion Gel Development}

Various viscosity imparting agents were evaluated for ME1, ME4 and ME5 formulations. The initial screening was conducted visually at room temperature over $24 \mathrm{~h}$. The results of this investigation are reported in Table 4. Carbopol 934 and Xanthan Gum were found to be compatible viscosity imparting agents for ME1 and ME4, whereas, ME5 was only stable with 
Colloidal Silica. The symbols used in Table 4 are explained below:

$\begin{array}{ll}\checkmark & \text { Stable microemulsion } \\ \mathrm{X} & \text { Incompatible } \\ \mathrm{N} / \mathrm{A} & \text { Not tested }\end{array}$

The physical stability of the Carbopol 934, Xanthan Gum and Colloidal Silica ME gels were then tested over 2 months at $40^{\circ} \mathrm{C}$ and using temperature cycling over 14 days. All formulations remained clear and isotropic, which indicated they were suitable for further development; however, it was observed that the viscosity of the ME5 Colloidal Silica gel increased after storing at $40^{\circ} \mathrm{C}$ for 3 weeks.

Table 4. Effect of viscosity imparting agents on microemulsions stability after $24 \mathrm{~h}$ at room temperature

\begin{tabular}{lccc}
\hline System & ME1 & ME4 & ME5 \\
\hline Lubrajel & $\mathrm{X}$ & $\mathrm{X}$ & $\mathrm{X}$ \\
Carbopol 934 & $\sqrt{ }$ & $\sqrt{ }$ & $\mathrm{X}$ \\
Xanthan Gum & $\sqrt{ }$ & $\sqrt{ }$ & $\mathrm{X}$ \\
HPMC K100M & $\mathrm{X}$ & $\mathrm{X}$ & $\mathrm{X}$ \\
Colloidal Silica & N/A & N/A & $\sqrt{ }$ \\
\hline
\end{tabular}

\section{Rheology of Microemulsion Gels}

The rheological profiles of various formulations indicated that the viscosity and elastic modulus of formulations decreased with application of thermal or mechanical stress for all the formulations except for ME5 thickened with Colloidal Silica. The rheological profiles are shown in Figure $2 \mathrm{~A}$ to $2 \mathrm{~F}$ and have been discussed further in following section.

\section{In vitro Release}

The in vitro release rates of Drug $\mathrm{X}$ from ME1, ME4 and ME5 gels were determined using diffusion cells. Figure 3 shows the plots of the cumulative amount of drug released per surface area (Q) over 6 hrs. The plot was used to calculate the in vitro release rates and permeation coefficients for each formulation, shown in Table 5. The ME4 with Xanthan Gum provided the best release rate followed by ME1 of Xanthan Gum and then ME5 gel.

\begin{tabular}{|c|c|}
\hline Formulation & $\begin{array}{c}\text { Release rate, } \\
J_{\mathrm{s}}=\left(\mu \mathrm{g} / \mathrm{cm}^{2}\right) / \mathbf{h}^{0.5}\end{array}$ \\
\hline ME1 + 0.5\% Xanthan Gum & 93.4 \\
\hline ME1 + 2\% Carbopol 934 & 11.5 \\
\hline ME4 + $0.3 \%$ Xanthan Gum & 127.7 \\
\hline ME4 + 1.2\% Carbopol 934 & 12.6 \\
\hline ME5 + 9\% Colloidal Silica & 88.5 \\
\hline Ointment (PG emulsion in oily phase) & 13.0 \\
\hline $\begin{array}{l}\text { The ointment and Carbopol } 934 \text { gels } \\
\text { release rates. }\end{array}$ & significantly lower \\
\hline
\end{tabular}

\section{DISCUSSION}

The objective of this study was to develop low surfactant ME gels for enhanced topical delivery of a poorly soluble model drug $\mathrm{X}$.

ME development was initiated by screening different surfactants, solvents and oily ingredients for solubility of drug X (Table 1). Five surfactant systems were then developed and ME regions of existence determined using pseudo-ternary phase diagrams (Figure 1).

These ME formulations were then evaluated visually for their physical stability at accelerated conditions and during temperature cycling. No signs of phase separation or turbidity were observed for the five formulations, indicating good physical stability. ME1, ME4 and ME5 were selected for further progression based on higher drug solubility of drug X (Table 3 ) in these formulations.

ME gel development involved screening of various viscosity imparting agents at room temperature over a $24 \mathrm{~h}$ period to obtain a rapid assessment and narrow down the number of gels for physical assessment. Carbopol 934 and Xanthan Gum gels of ME1 and ME4, and a ME5 Colloidal Silica gel, were the only stable ME gels (Table 4). These formulations were also stable at accelerated conditions and during temperature cycling.

The behaviour and stability of these formulations during high mechanical stress conditions, such as filling or application and spreading onto skin, were tested using the dynamic stress ramp test at $25^{\circ} \mathrm{C}$ and $32^{\circ} \mathrm{C}$ (Figure $2 \mathrm{~A}$ and B). 


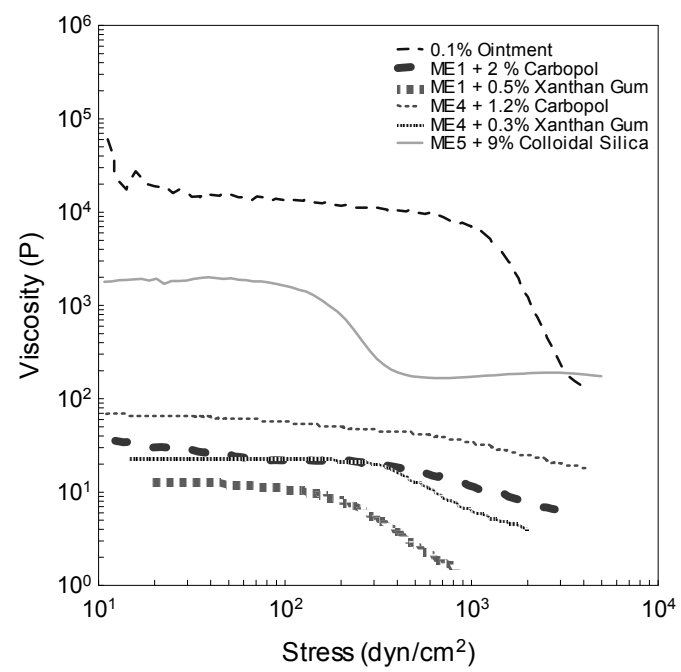

A: Viscosity versus stress at $25^{\circ} \mathrm{C}$

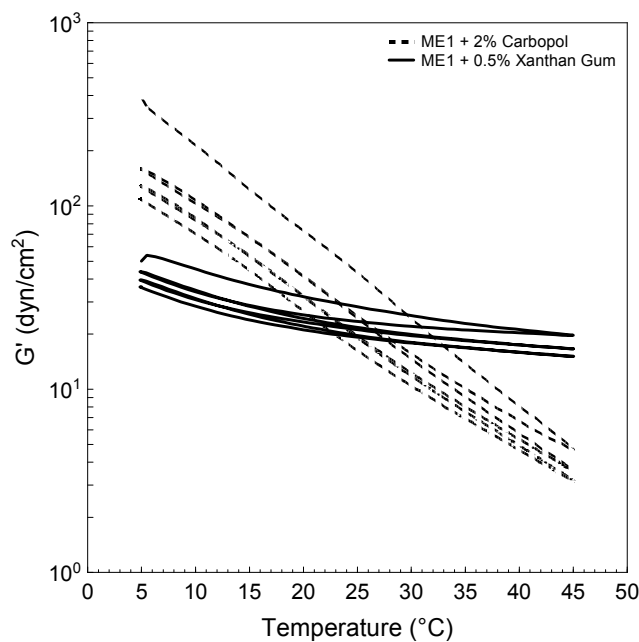

C: $\mathrm{G}^{\prime}$ vs. temperature for $\mathrm{ME} 1$

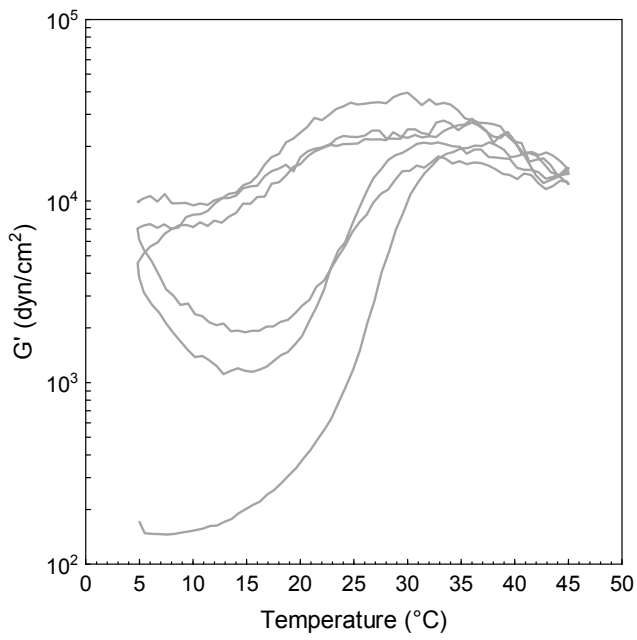

E: $G^{\prime}$ vs. temperature for ME5

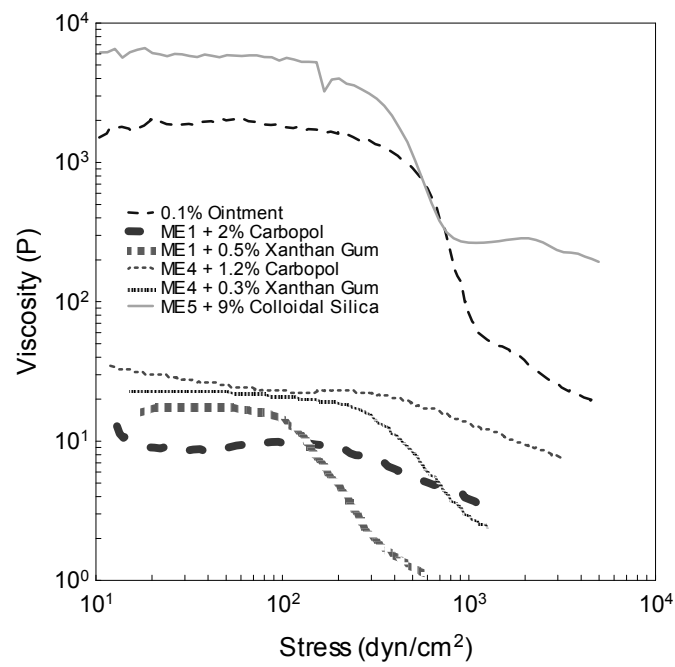

B: Viscosity versus stress at $32^{\circ} \mathrm{C}$

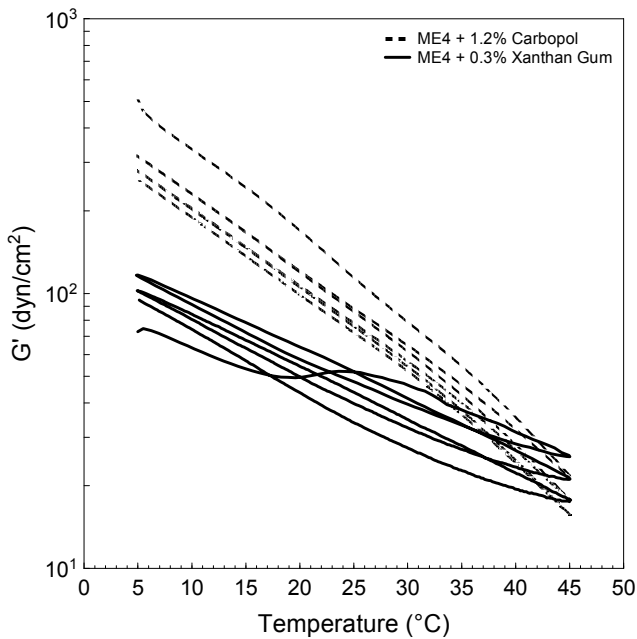

D: $\mathrm{G}^{\prime}$ vs. temperature for ME4

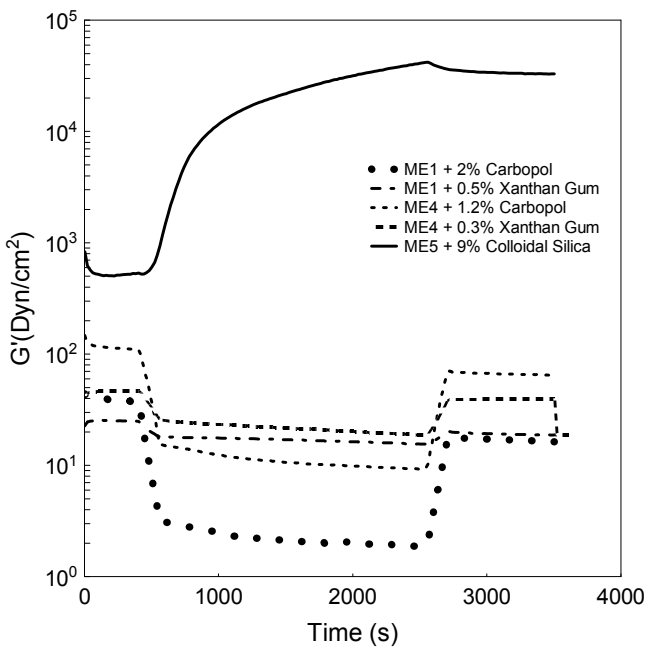

F: Temperature step test

Figure 2. Rheological tests for microemulsion gels. Dynamic stress sweep test at $25^{\circ} \mathrm{C}(\mathrm{A})$ and $32^{\circ} \mathrm{C}(\mathrm{B})$, temperature ramp cycle tests for ME1 (C), ME4 (D) and ME5 (E), and temperature step test (F). 


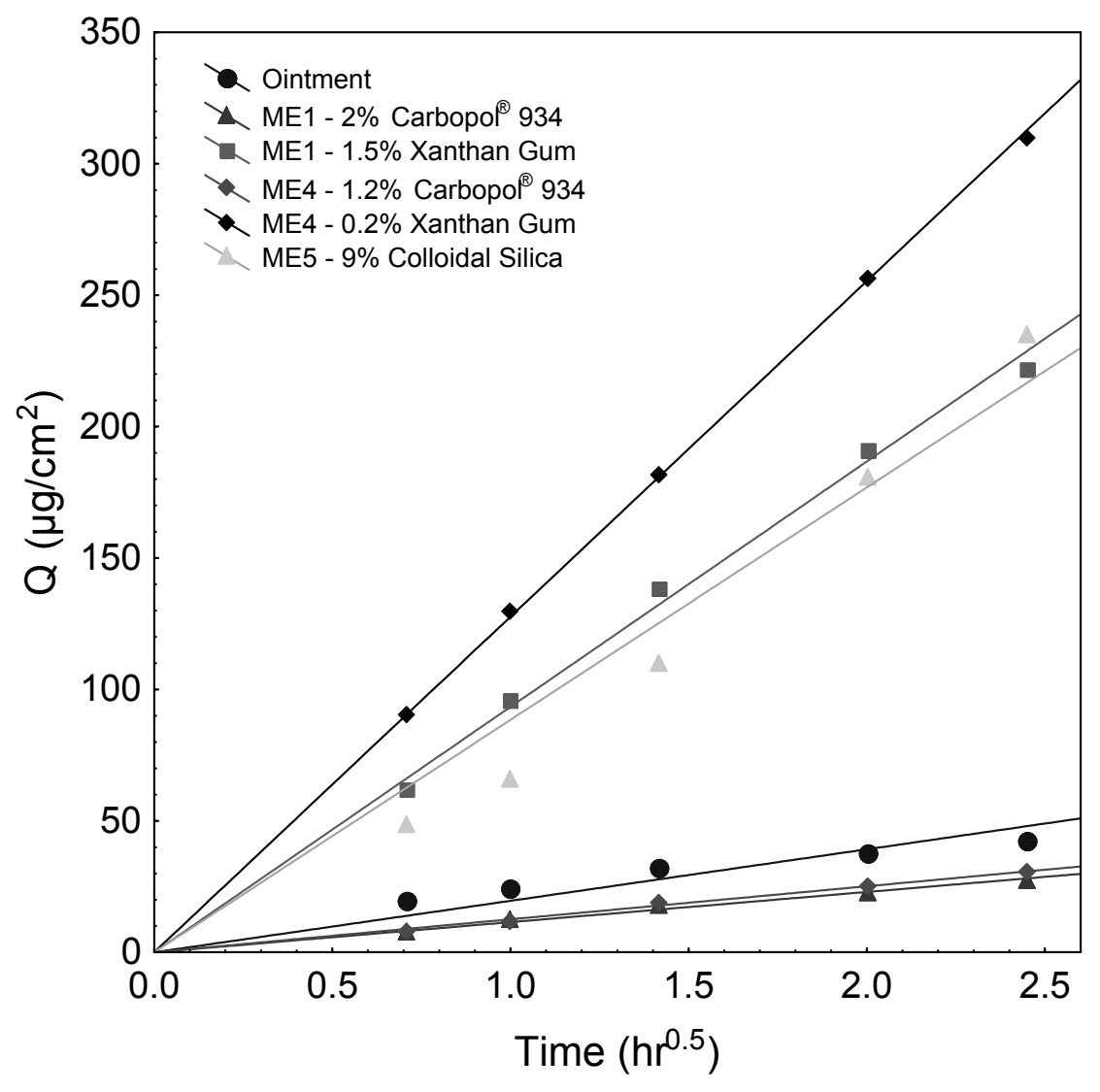

Figure 3. Release rate of Drug $X$ from various formulations $(n=2)$

Overall, the tests showed that the ME gels had lower viscosities compared to conventional ointment, indicating that the gels would behave well during manufacture and application. For ME gels made from Xanthan Gum and Colloidal Silica, well defined yield stress values were observed, indicating rapid drops in viscosity at high shear stress. Carbopol 934 gels did not have well defined yield stress values but did show a gradual decrease in viscosity with increase in stress. Had the viscosity of the Xanthan Gum and Carbopol 934 gels been higher, the shear thinning properties of the ME Xanthan Gum gels would indicate behaviour better suited for manufacture and application to the skin.

Xanthan Gum and Carbopol 934 gels showed reduced viscosity at skin temperature $\left(32^{\circ} \mathrm{C}\right)$ supporting the conclusion that these gels would perform well in topical applications. The increase in viscosity observed for ME5 Colloidal Silica gel would adversely impact application.
The hysteresis loops generated by studying the change in the elastic modulus $\left(\mathrm{G}^{\prime}\right)$ with temperature (Figure $2 \mathrm{C}-\mathrm{E}$ ), provide a measure of structural changes that take place in the formulation on application of stress. Small hysteresis loops indicate smaller structural changes with temperature and hence greater physical stability (12). ME1 and ME4 formulations thickened with Xanthan Gum and Carbopol 934 showed very narrow hysteresis loops (Figure $2 \mathrm{C}$ and $\mathrm{D}$ ), indicating good stability of these formulations. The slight decrease in the value of $\mathrm{G}^{\prime}$ in subsequent loops for the Xanthan Gum and Carbopol 934 gels indicates thixotropy or shear thinning nature of these formulations. For the ME4 gels, the Carbopol 934 gel had smaller loops which indicated better stability compared to the Xanthan Gum gel (Figure 2D). The Xanthan Gum gel, however, showed less sensitivity to temperature change than Carbopol 934 gels in both the formulations. The hysteresis loops for Colloidal Silica gel of ME5 show that the elastic behaviour of 
this formulation increases with temperature, which is different from the Xanthan Gum and Carbopol 934 gels. In addition, large change in $\mathrm{G}^{\prime}$ and bigger hysteresis loops suggests a major structural change or instability of the formulation with changes in temperature (Figure $2 \mathrm{~F}$ ).

A temperature step test was used to determine the responses of the formulations to large temperature changes that can be encountered during storage or transportation (Figure $2 \mathrm{D}$ ). At $25^{\circ} \mathrm{C}$, all the formulations appear to be stable. Xanthan Gum gel of ME1 and colloidal Silica gel of ME5 have lowest and highest $\mathrm{G}^{\prime}$ value, respectively. When the temperature was increased to $50^{\circ} \mathrm{C}$, Xanthan Gum gels of ME1 and ME4 showed excellent thermal stability which is evident from the minimal change in $\mathrm{G}^{\prime}$ value and almost complete recovery to their original $G^{\prime}$ values when the thermal stress was removed. Carbopol 934 gel of ME1 and ME4 showed less thermal stability compared to Xanthan Gum gels since there is a larger drop in $\mathrm{G}^{\prime}$ with change in temperature and the $G^{\prime}$ value was not completely recovered when the thermal stress was removed. In ME5, the elastic modulus increased sharply with increase in temperature before reaching a constant value. The value of $\mathrm{G}^{\prime}$ did not drop after the thermal stress was removed indicating some irreversible structural change occurring in the formulation at $50^{\circ} \mathrm{C}$. Together with the temperature ramping data, these results predict that ME5 irreversibly loses its processability and spreadability when exposed to high temperatures. This prediction is in line with the results from accelerated testing that showed the viscosity of the ME5 Colloidal Silica gel increased after being stored at $40^{\circ} \mathrm{C}$ for 3 weeks. From all the rheological investigations it can be concluded that Xanthan Gum formulations of ME1 and ME4 demonstrated the best rheological profiles amongst all the formulations tested.

In vitro release testing indicated that ME4 containing Xanthan Gum showed the best release rate $\left(\mathrm{J}_{\mathrm{s}}=127.7 \mu \mathrm{g} / \mathrm{cm}^{2} / \mathrm{h}^{0.5}\right)$ followed closely by Xanthan Gum gel of ME1 $\left(\mathrm{J}_{\mathrm{s}}=93.4 \mu \mathrm{g} / \mathrm{cm}^{2} / \mathrm{h}^{0.5}\right)$, and ME5 thickened with colloidal silica $\left(\mathrm{J}_{\mathrm{s}}=\right.$ $\left.88.5 \mu \mathrm{g} / \mathrm{cm}^{2} / \mathrm{h}^{0.5}\right)$. The release rates from the ME gels containing Xanthan Gum and colloidal silica were 8 to 10 fold higher than those from conventional ointments and formulations containing Carbopol 934.
There are three possible explanations for this higher flux rate. First, the higher solubility of the drug in ME gels compared to ointment is possibly a significant factor in increasing the drug release rate as only the dissolved fraction of a drug in a vehicle can cross the membrane. Second, at room temperature, $\mathrm{ME}$ gels have lower viscosity $\left(10^{1}\right.$ $\left.10^{3} \mathrm{P}\right)$ compared to ointment $\left(10^{5} \mathrm{P}\right)$. The lower viscosity results in better drug motility in the vehicle which improves drug flux across the membrane (13). However, this statement does not hold good for ME5 thickened with Colloidal Silica. Although the viscosity of this formulation at $32^{\circ} \mathrm{C}$ (Figure 2B) is higher than that of the conventional ointment, the release rate of former is much higher. Third, ME structural organization might play a role in enhancing drug transport across the membrane. Small droplet size coupled with low interfacial tension due to high surfactant concentration could potentially improve drug diffusion across the membrane.

The choice of thickening agent has an important effect on release rate of drug from ME gels. Use of Carbopol 934 substantially lowered the release rate (approximately 10 fold) of the model drug compared to ME gels containing Xanthan Gum. It is postulated that Carbopol 934 polymer hinders the drug release by entrapping the drug into its complex structure or by forming chemical interactions with the drug. It is to be noted that due to considerable differences between receptor media versus body fluids and artificial membrane versus skin, the release rates shown above may be different than those obtained from actual in vitro skin flux studies. Nevertheless, these results can be used to show the superior performance of the ME gels over conventional formulations.

ME4 gel with Xanthan Gum performed the best amongst all the formulations tested. It provided the highest rate of release and best physical stability at accelerated conditions.

\section{CONCLUSIONS}

Ease of manufacturing, improved solubility and favourable cutaneous drug delivery, together with very good physical stability, make MEs attractive drug delivery systems. In this study we explored the possibility of utilizing low surfactant MEs as topical formulations for a poorly water soluble drug using compendial grade excipients. 
The model drug showed improved solubility (up to 14 fold over conventional ointment) in selected MEs. In vitro release profiles revealed that ME gels can substantially improve the drug release compared to conventional ointment formulation. All the formulations exhibited excellent physical stability, i.e. no separation or turbidity was observed during accelerated studies. Xanthan Gum and Carbopol 934 were found to be good viscosity imparting agents although formulations thickened with Carbopol 934 showed significantly lower release rates compared to those with Xanthan Gum. Rheological assessments of the gels proved that Xanthan Gum has the best profile for ease of application and processability. Both Xanthan Gum and Carbopol 934 gels showed acceptable thermal stability in temperature ramp cycle test and temperature step test. Formulations thickened with Colloidal Silica showed irreversible thickening of formulation on application of thermal stress, which is not desirable for topical formulations. It is evident that the choice of thickening agent plays an important role in governing the drug release from ME gels as formulations thickened with Carbopol 934 showed significant decrease in their drug release rate. Despite better stability, ease of manufacture and higher release of drug from these ME gels, their irritation potential still needs to be investigated.

ME4 gel with Xanthan gum was found to be the best formulation and this may be progressed for further product development.

\section{REFERENCES}

1. Danielson I, Lindman B. The definition of microemulsion. Colloid. Surf. 1981; 3: 391-392.

2. Kreilgaard M. Influence of microemulsions on cutaneous drug delivery. Adv. Drug Deliv. Rev. 2002; 54 Suppl. 1: 77-98.

3. Shinoda K, Lindman B. Organized surfactant systems: microemulsions, Langmuir. 1987; 3: 135149.

4. Malcolmson C, Lawrence M.J. A comparison between nonionic micelles and microemulsions as a means of incorporating the poorly water soluble drug diazepam. J. Pharm. Pharmacol. 1990; Suppl. 42:6.

5. Malcolmson C, Satra S. Kantaria A, M.J. Lawrence. Effect of oil on the level of solubilization of testosterone propionate into nonionic oil-in-water microemulsions. J. Pharm. Sci. 1998; 87: 109-116.
6. Goldberg-Cettina M, Liu P, Nightingale J. Enhanced transdermal delivery of estradiol in vitro using binary vehicles of isopropyl Myristate and short chain alkanols. Int. J. Pharm. 1995; 114: 237-245.

7. Peltola S, Saarinen-Savolainen P, Kiesvaara J, Suhonen M, Urtii A. Microemulsions for topical delivery of estradiol. Int. J. Pharm. 2003; 254: 99107.

8. Spiclin P, Homar M, Zupancic-Valant A, Gasperlin M. Sodium ascorbyl phosphate in topical microemulsions. Int. J. Pharm. 2003; 256: 65-73.

9. Osman-Gardabbou H, Pelletier J, Sfor-Gandoura S, et al. Thickening of hydrophilic/lipophilic and lipophilic/hydrophilic microemulsions. Characteristics of the $\mathrm{H} / \mathrm{L}$ and $\mathrm{L} / \mathrm{H}$ microemulsions. STP. Pharm. Sci. 10: 220-223.

10. Osman-Gardabbou H, Pelletier J, Sfor-Gandoura S, et al. Thickening of hydrophilic/lipophilic and lipophilic/hydrophilic microemulsions. Comparative study of the thickening influence on $\mathrm{H} / \mathrm{L}$ and $\mathrm{L} / \mathrm{H}$ microemulsions as enhancers for lipophilic tracer. STP. Pharm. Sci. 10: 224-228.

11. Ceschal G.C, Maffei P, Moretti M.D, Demontis S, et al. In vitro permeation through porcine buccal mucosa of salvia desoleana atzei and picci essential oil from topical formulations. Int. J. Pharm. 2000; 195: 171-177.

12. Yao M, Patel J. Rheological characterization of body lotions. Rheometric Scientific Inc.

13. Istvan Eros, Ehab Youseff Abu-Elida, Ildiko Csoka, Zsuzsa, Annamaria Cserne and Tunde Kover. Optimization of drug release from dermatological semisolids preparations. Drug Development Research. 2003: 59: 316-325.

14. Warisnoicharoen W, Lansley A. B, Lawrence M. J. Nonionic oil-in-water microemulsions: the effect of oil type on phase behaviour. Int. J. Pharm; 2000, 198: 7-27. 